\title{
Contratos de riesgo de petróleo, patrones de conducta y desempeño de empresas: Análisis de panel de datos
}

\author{
Oil risk contracts, business conduct and performance patterns: \\ Data panel analysis
}

\author{
Alejandro Ibarra Yúnez ${ }^{a, *}$ y Fidel Flores Chapa ${ }^{b}$ \\ a Egade Business School, Tecnológico de Monterrey, México \\ ${ }^{\mathrm{b}}$ Escuela de Ingeniería y Ciencias, Tecnológico de Monterrey, México \\ Recibido el 21 de junio de 2016; aceptado el 16 de febrero de 2017 \\ Disponible en Internet el 1 de noviembre de 2017
}

\section{Resumen}

Usando datos del desempeño económico-financiero de las compañías internacionales de exploración y exploración-extracción de petróleo o IOC, así como los patrones de situación institucional y orientación con el mercado de gobiernos y empresas nacionales de petróleo o NOC receptoras de ofertas de proyectos, se analiza el desarrollo institucional y los patrones de conducta por tipo de contrato de compañías de exploraciónextracción, siguiendo el enfoque de actor estratégico, o la llamada agency theory. Adicionalmente, ante la reforma energética de México, en proceso de implementación entre 2015 y 2019, se analizan los tipos de contratos de licencia frente a los de producción y utilidad compartida. Luego, utilizando métodos de panel de datos en análisis de 17 empresas entre 2005-2015, se determina que las empresas llamadas globales presentan rendimientos y compromisos mayores frente a las especializadas, demostrados por sus ingresos netos y rendimientos sobre capital o ROE.

(C) 2017 Universidad Nacional Autónoma de México, Facultad de Contaduría y Administración. Este es un artículo Open Access bajo la licencia CC BY-NC-ND (http://creativecommons.org/licenses/by-nc-nd/4.0/).

Palabras clave: Economía de las regulaciones; Modelos de negociación; Exploración de petróleo; Modelos de datos en panelL51, C78, L71, C23

\footnotetext{
* Autor para correspondencia.

Correo electrónico: aibarra@itesm.mx (A. Ibarra Yúnez).

La revisión por pares es responsabilidad de la Universidad Nacional Autónoma de México.
} 


\begin{abstract}
Using data from financial and economic performance of International Oil Companies (IOCs) in the exploration-extraction (E\&E) business, along with institutional and market orientation of governments and National Oil Companies (NOCs) that receive project offers, the international E\&E market is analyzed in both institutional development and behavioral patterns of type of E\&E contract following an agency theory approach. Additionally, given Mexico's recent energy reform being launched between 2015 and next 2019, the various types of E\&E contracts are analyzed, comparing license contracts with production sharing and risk service ones. Next, using panel data methods an analysis of 17 enterprises between 2005 and 2015 is presented, where so-called global IOCs show higher returns and commitment than specialized ones, demonstrated by their net income and return on equity, or ROE.

(C) 2017 Universidad Nacional Autónoma de México, Facultad de Contaduría y Administración. This is an open access article under the CC BY-NC-ND license (http://creativecommons.org/licenses/by-nc-nd/4.0/).
\end{abstract}

Keywords: Economics of regulation; Bargaining models; Oil exploration; Panel data models

JEL classification: L51, C78, L71, C23

\title{
Introducción
}

Varios países petroleros han abierto su sector de exploración y extracción de petróleo (E\&E) a la inversión de las empresas contratistas globales o internacionales, llamadas en inglés IOC ${ }^{1}$, a través de diversos tipos de acuerdos (Al-Attar y Alomair, 2005), tal como lo hizo México con la reforma energética (RE) en 2014, después de casi 80 años de depender de su empresa estatal Pemex e invertir capital nacional para el desarrollo del sector.

El enfoque de desregulación y apertura a la iniciativa privada responde a la necesidad de los gobiernos de compartir los riesgos de inversión (Feng, Zang y Gao, 2014), y al mismo tiempo fortalecer el sector con la transferencia de tecnología, experiencia y capital de las empresas contratistas IOC, ya sea solas o en coinversión con empresas privadas nacionales o POC. En años recientes la mayoría de los países que mantienen nacionalizado su sector petrolero han disminuido su producción y desacelerado la exploración, debido a la falta de tecnología pertinente y experiencia, así como a las limitaciones institucionales y políticas (Al-Attar y Alomair, 2005), sobre todo con efectos en restricciones de inversión. Con ello, la razón económica parece ser fundamental para entender las acciones de reformas regulatorias y el alistamiento de empresas globales en E\&E.

Las limitaciones de la empresa paraestatal reformada Pemex, ahora empresa productiva del Estado, están presentes en México, donde los ingresos del Estado dependen de manera importante de los ingresos petroleros, y debido a la necesidad atender otros sectores se invierte menos de lo que requiere la prospección de hidrocarburos para el aseguramiento de altos niveles de producción en el futuro. Esta falta de capital se pretende subsanar mediante la participación de las IOC y las POC.

El decreto constitucional de la RE indica que para llevar a cabo las actividades de E\&E de hidrocarburos sólidos, líquidos o gaseosos, incluyendo las actividades que puedan realizar las

\footnotetext{
${ }^{1}$ En este artículo, cuando se hable de las IOC, deberá entenderse que el término se refiere a cualquier persona moral con posibilidad de ser contratista, por lo cual el concepto es amplio e incluye también empresas privadas nacionales o POC, así como subcontratos de tipo coinversión, nacionales o extranjeros.
} 
empresas del Estado con particulares, deberán ser a través de contratos en términos de lo dispuesto por el artículo 27 de la Constitución. El decreto señala también que se prohíbe el otorgamiento de concesiones para este sector (D.O.F., 2013), esto en el interés del Estado de mantener la soberanía de los recursos y garantizar el control y la observancia de las actividades petroleras.

El mismo decreto establece que los contratos para E\&E de petróleo podrán ser: a) contratos de servicios; b) contratos de utilidad compartida; c) contratos de producción compartida (PSC); d) contratos de licencia; y e) cualquier combinación de los anteriores. En cada caso, el Estado definirá el modelo contractual que mejor convenga para maximizar los ingresos del país. La ley establecerá las modalidades de las contraprestaciones que pagará el Estado a sus empresas productivas o a los particulares por las actividades de E\&E del petróleo y de los demás hidrocarburos.

Entre otras modalidades de contraprestaciones se regulan las siguientes: a) en efectivo, para los contratos de servicios; b) con un porcentaje de la utilidad, para los contratos de utilidad compartida; c) con un porcentaje de la producción, para los contratos de producción compartida; d) con la transmisión onerosa de los hidrocarburos una vez que hayan sido extraídos del subsuelo, para los contratos de licencia; y e) cualquier combinación de las anteriores.

Existe un reto especialmente crítico para países que han desregulado su sector energético. El presente estudio busca dar una base tanto teórica como empírica sobre los contratos de petróleo de E\&E con una empresa establecida o incumbente, ahora definida como empresa productiva del Estado, Pemex, y con participantes privados que cuentan con capacidad de inversión y con capacidad tecnológica, tanto de empresas privadas nacionales como empresas globales con varios tipos de especialización productiva en negocios petroleros, o IOC, algo nuevo para México. El análisis primero tipifica los patrones de conducta de las empresas contratistas de manera teórica, basado en preferencias de las mismas y los gobiernos anfitriones, para luego vincular empíricamente su desempeño medido en ingresos netos y ROE, con los patrones de conducta derivados de IOC globales frente a especializadas.

De acuerdo con Jaffe y Soligo (2007) aproximadamente un 33\% de todos los contratos de E\&E en el mundo son entre un gobierno anfitrión (Host Government [HG]) y su NOC, con la respectiva IOC. Es decir, el mercado de contratos internacionales se caracteriza por mercados concentrados. Por ello, el análisis se justifica en términos de actores estratégicos con enfoque de teoría de la agencia.

El estudio está dividido de la siguiente manera: en la siguiente sección se revisa la literatura relevante sobre contratos de E\&E desde la perspectiva del actor estratégico; la tercera parte se enfoca a plantear el marco de referencia de los contratos, desde la unión de los enfoques de motivaciones explícitas de los gobiernos y las IOC contratistas, y se desarrollan los modelos teóricos de contratos de producción compartida, licencias y servicios de riesgo con recompra, así como los equilibrios para gobiernos frente a contratistas por tipo de contrato óptimo. La cuarta sección usa los datos de las empresas IOC y los tipos de gobiernos para derivar con pooling de datos y análisis de paneles, la validación de los modelos algebraicos de la sección previa. En esta sección empírica se comprueba el desempeño económico (ingresos netos de las empresas) y financiero (ROE), con los tipos de empresas globales frente a especializadas en sus patrones de conducta. La quinta sección concluye y deriva implicaciones para México.

\section{Literatura relevante sobre contratos internacionales de petróleo}

La literatura sobre contratos de E\&E de petróleo es escasa, dado que muchos contratos entre gobiernos y NOC, con contratistas internacionales o grupos privados, tradicionalmente se han realizado de manera bilateral, con poca transparencia y donde las NOC no son empresas públicas 
listadas en bolsa. Por su parte, las contratistas IOC, al no ser empresas públicas o desarrollar proyectos públicos, en muchos casos no difunden su información financiera o de los proyectos mismos. Por ello, existen iniciativas de grupos no gubernamentales, como la Extractive Industry Transparency Initiative, que busca desde 2003 hacer pública la información de todos los contratos y tratos fiscales y de regalías de los proyectos extractivos del mundo, que sin embargo, solo cuenta con un grupo de cerca de 40 países miembros: los EE. UU., la Unión Europea, Noruega, varios países de África, así como Kazakstán, Kirguistán, Tayikistán y algunos países de Asia (EITI, 2016).

A pesar de lo anterior, el modelaje de contratos internacionales de E\&E, en términos de teoría de juegos y optimización, ha sido planteada en trabajos de Al-Attar and Alomair (2005) sobre alternativas contractuales para los NOC frente a la empresa contratista; Feng et al., 2014 y Ghandi y Lin (2014) realizan comparaciones entre países destino de acuerdo con los activos estratégicos vistos por las contratistas; Jenik (2005) se concentra en la parte fiscal y Johnston (2003) y Van Groenendaal y Mazraati (2006) utilizan modelos contractuales más completos. Todos los anteriores trabajos parten de la base de Laffont sobre las funciones objetivo de los gobiernos (bienestar con o sin impuestos) frente a las empresas reguladas que maximizan utilidades, tomando como endógenas las variables de selección adversa y esfuerzo reductor de costos, ya que son variables no observadas por el gobierno o regulador (Laffont, 2005).

\section{Contratos de exploración-explotación: enfoque de agentes-principales}

\section{Motivaciones de los jugadores en sus objetivos}

Las concesiones y los contratos petroleros son instrumentos jurídicos bilaterales mediante los cuales se establecen derechos y obligaciones de la empresa o grupo de empresas (contratista) y del Estado (contratante). Existen distintos esquemas contractuales utilizados en todo el mundo por los gobiernos anfitriones para permitir que las IOC lleven a cabo exploraciones de petróleo y, en el caso de un descubrimiento comercial, inviertan en el desarrollo y explotación del campo petrolero. Estos tipos de contrato han sido analizados por la teoría económica, la teoría institucional, la teoría de estrategia y por la teoría de juegos y optimizaciones binivel.

Un sistema fiscal petrolero se refiere a los términos y las condiciones establecidos en un contrato de común acuerdo entre el gobierno y la IOC. Se compone de pagos realizados por concepto de impuestos y derechos que tiene la IOC o POC y el Estado (por lo general a través de su NOC) y que implica obligaciones para ambas partes. Para diseñar un sistema fiscal los países consideran el marco legal relacionado con los recursos petroleros, las estrategias de desarrollo, las actividades a estimular y las experiencias obtenidas, por lo que cada régimen posee características que lo diferencian del resto. Isehunwa y Uzoalor (2011) definen los regímenes fiscales como el marco para administrar, regular y compartir los ingresos del petróleo, por ello son importantes para el gobierno anfitrión llamado en la literatura HG y las IOC.

De acuerdo con Jenik (2005) los gobiernos de los países productores tienen básicamente 3 maneras de poder aprovechar sus recursos fósiles:

a) A través de una empresa estatal, que pueda llevar a cabo la E\&E de hidrocarburos y en algunos casos todas las actividades de la cadena productiva del sector (empresa integrada verticalmente). Tal es el caso de países como Arabia Saudita, Venezuela e Irán. 
b) Por medio de inversión privada, empleando múltiples empresas especializadas en actividades específicas y/o empresas petroleras integradas, como en Estados Unidos, Rusia, Reino Unido y Canadá.

c) Mediante la combinación de los 2 sistemas anteriores, como es el caso de Indonesia, Nigeria, Azerbaiyán, Kazakstán y desde la RE México.

Al-Attar y Alomair (2005) dividen la historia de los acuerdos petroleros en 3 eras principales. Primero fue la edad de las concesiones, cuando las IOC dominaron el sector del petróleo a nivel mundial, a través de acuerdos de concesión clásicos en diferentes partes del mundo. Posteriormente se vivió la era del nacionalismo económico, cuando proliferó la creación de las empresas petroleras del estado o NOC, así como la aparición de nuevos tipos de acuerdos de petróleo. Finalmente, está la época actual en que los países han reabierto sus sectores de «aguas arriba» o upstream de petróleo a la inversión privada.

Por su parte Johnston (2003) plantea que los regímenes fiscales de petróleo se pueden dividir en 2 tipos en función de la propiedad del petróleo: sistemas basados en la imposición de regalías e impuestos (concesiones), donde la IOC tiene la propiedad del petróleo, y sistemas de contratos donde el Estado mantiene la soberanía de los recursos.

El sistema contractual se subdivide a su vez de acuerdo con el tipo de contrato: contratos de servicios y contratos de PSC. A su vez, los contratos de servicios pueden ser de 2 tipos: contratos de servicios puros y contratos de servicios de riesgo, en términos de la asignación de riesgos entre el gobierno anfitrión y la IOC. Además, existen los contratos de recompra, Buy-Back Contract o Buy-Back Agreement (BBA), que son un tipo particular de contratos de servicios de riesgo (Van Groenendaal y Mazraati, 2006). Otro acuerdo que podría incluirse es el contrato de licencia ${ }^{2}$ (LC).

De acuerdo con Le Leuch (1988) el modelo de contrato elegido por un gobierno, así como los términos y condiciones acordados entre las partes firmantes, dependen principalmente de las políticas del país anfitrión y de las capacidades de negociación de sus gobiernos. Al mismo tiempo están directamente relacionados con el potencial de explotación de la superficie ofrecida y la situación del mercado internacional de petróleo. Adicionalmente, ya que la contratista (IOC/POC) tiene en las variables de «esfuerzo» y selección adversa como información privada, se convierte en el agente o «actor estratégico» frente al HG que juega como el «principal» (King y Spalding, 2005).

Por otra parte, los costos totales de E\&E, así como el factor de recuperación, son 2 principales factores que determinan el tipo de contrato petrolero que el HG adoptará. Por ejemplo, en los países con reservas relativamente bajas y altos costos de producción debido a que el petróleo se encuentra en aguas ultra profundas, como el Reino Unido, los Estados Unidos y Noruega, el sistema dominante es el de regalías e impuestos. En contraste, cuando las reservas son grandes y los costos son medianos, como en Kazajstán, Omán, China, Indonesia y Nigeria, los acuerdos de producción compartida son más frecuentes. Mientras tanto, los contratos de servicios puros y de riesgo tienden a ser utilizados en países con grandes reservas y bajos costos de E\&E, como en Kuwait, Irán y Venezuela.

No obstante, algunos países como México han adoptado más de un tipo de acuerdo de petróleo, ya que tienen tipos de reservas de petróleo diferenciados, con un amplio rango de costos de E\&E, así como que en un juego de información asimétrica es lógico que el gobierno anfitrión ofrezca un

\footnotetext{
${ }^{2}$ Los contratos de licencia son un tipo de contrato particular que equivale en sus términos a una combinación entre una concesión moderna y un contrato de producción compartida.
} 
portafolio de tipos de contrato, para que las IOC se autoseleccionen, de acuerdo con sus patrones de conducta sobre aceptación o aversión al riesgo y su característica como empresas globales o especializadas en alguna parte de la E\&E (constructoras, transportistas, especializadas en aguas profundas, etc.).

Más aún, ya que los objetivos primordiales de los HG son maximizar la riqueza de sus recursos fósiles (Blake y Roberts, 2006) y tener control de la actividad petrolera, recurren al establecimiento de compromisos de trabajo y determinados sistemas fiscales (Ashong, 2010). Por ejemplo, se realizan pagos no únicos, argumentativamente incentivados, a través de bonos a la firma del contrato, y durante la producción, mediante regalías, impuestos y la participación en la producción o en las utilidades. Por otra parte, la IOC busca la maximización de su riqueza mediante la búsqueda de reservas de petróleo y la producción de hidrocarburos con el menor costo y el más alto margen de beneficio posible, en el menor tiempo (Johnston, 1994).

De acuerdo con Grunstein (2010) y Feng et al. (2014), los intereses que se pueden ver reflejados en los contratos responden como mínimo a lo siguiente:

Por el lado de los gobiernos anfitriones (HGs):

- Maximización de la renta petrolera.

- Incremento de las reservas nacionales.

- Control de las operaciones mediante compromisos de trabajo.

- Aseguramiento del abasto nacional (seguridad energética).

- Desarrollo tecnológico de la industria y contenido nacional.

- Protección al medio ambiente y garantía de existencia de reservas ante accidentes.

Por el lado de los contratistas (IOCs/POCs):

- Proporcionalidad entre el riesgo del proyecto y compensación.

- Maximización de las ganancias.

- Contabilización de las reservas.

- Flexibilidad contractual y estabilización regulatoria.

- Recuperación de costos de inversión.

- Menos control administrativo del HG sobre el contrato.

- Capacidad de arbitraje internacional.

\section{Planteamiento de los modelos teóricos de conducta}

Con la elaboración de los modelos mencionados de Feng, Zhang y Gao (2014), así como de Laffont (2005), podrían desarrollarse los modelos alternativos como sigue. Compárense las características de cada tipo de contrato, empezando por el PSC, pasando luego a plantear el LC y adicionando luego para contrastar y derivar aquellos teóricamente preferibles, el de BBA.

\section{Producción compartida}

Sea 1 el ingreso bruto $I_{B}$ definido como el volumen extraído Q por su precio P, ambas variables esperadas:

$$
I_{B}=P Q
$$


El monto por regalías ${ }^{3} \mathrm{R}$ es el producto del porcentaje de regalías $\alpha$ por el ingreso bruto.

$$
R=\alpha I_{B}
$$

Sustituyendo la ecuación 1 en la ecuación 2:

$$
R=\alpha P Q
$$

La cuota de recuperación de costos $C R$ es el porcentaje $\sigma$ del límite de recuperación de costos, por el remanente del ingreso bruto una vez restadas las regalías, ofrecida por el HG y calculada dentro de viabilidad por el agente IOC (cost oil):

$$
C R=\sigma\left(I_{B}-R\right)
$$

Sustituyendo las ecuaciones 1 y 3 en la ecuación 4 se obtiene la siguiente:

$$
C R=\sigma(1-\alpha) P Q
$$

La utilidad operativa total $U O_{T}$ es el resultado de restar el porcentaje de remanente de las regalías y el porcentaje de la recuperación de costos (ecuación 4) al ingreso bruto:

$$
U O_{T}=I_{B}-R-C R
$$

Sustituyendo las ecuaciones 1,3 y 5 en 6 , se obtiene:

$$
U O_{T}=(1-\sigma)(1-\alpha) P Q
$$

Ahora el monto de la utilidad operativa para la IOC $U O_{I O C}$ se obtendrá multiplicando el porcentaje de reparto $\delta$ por la utilidad operativa total.

$$
U O_{I O C}=\delta U O_{T}
$$

Sustituyendo la ecuación 7 en 8 , se obtiene:

$$
U O_{I O C}=\delta(1-\sigma)(1-\alpha) P Q
$$

La tasa de reparto $(\delta)$ es variable y sujeta de negociación entre el HG o principal, y el agente estratégico IOC. Por su parte, la IOC debe pagar una tasa de impuestos $\mu$ sobre su utilidad operativa.

$$
T_{P S C}=\mu U O_{I O C}
$$

Sustituyendo la ecuación 9 en la ecuación 10 se obtiene la tasa fiscal de impuestos, en términos de los otros porcentajes de los ingresos brutos:

$$
T_{P S C}=\mu \delta(1-\sigma)(1-\alpha) P Q
$$

El ingreso total de la IOC $I_{I O C}$ después de impuestos es:

$$
I_{I O C, P S C}=U O_{I O C}+C R-T_{P S C} .
$$

\footnotetext{
${ }^{3}$ En el caso de México el porcentaje de regalías es distinto para cada tipo de hidrocarburo y depende de los precios de cada uno. Para efectos de simplificación del modelo se sigue el planteamiento de Feng et al. (2014), tal que el porcentaje de regalías es el mismo para todos los productos y se aplica directamente al ingreso bruto, en concordancia con la práctica internacional.
} 
Sustituyendo las ecuaciones 5, 9 y 11 en la ecuación 12 anterior, resulta en:

$$
I_{I O C, P S C}=(1-\alpha)[\delta(1-\mu)(1-\sigma)+\sigma] P Q
$$

La cuota de recuperación de costos tiene la finalidad de sufragar los gastos operativos $O P E X$. Como la IOC asume estos gastos, la utilidad neta de la IOC $U_{I O C}$ se reduce a:

$$
* U_{I O C, P S C}=(1-\alpha)[\delta(1-\mu)(1-\sigma)+\sigma] P Q-O P E X
$$

Tómese la anterior ecuación como el primer tipo de equilibrio de este contrato PSC, y denótese con un asterisco $(*)$

\section{Contratos de licencia}

El ingreso bruto $I_{B}$ y el monto por regalías R son calculados igual que en un PSC (ecuaciones 1 y 3), es decir, al ingreso bruto se restan las regalías y de ese remanente un porcentaje $\omega$ le toca al HG, que corresponderá a la ganancia del HG $G_{H G}$

$$
G_{H G}=\omega\left(I_{B}-R\right)
$$

Sustituyendo las ecuaciones 1 y 3 en 15 se obtiene la siguiente:

$$
G_{H G}=\omega(1-\alpha) P Q
$$

La IOC recibe la transmisión onerosa de los hidrocarburos una vez pagadas las contraprestaciones del HG. Por lo tanto, el ingreso total de la IOC $I_{I O C, L C}$ es:

$$
I_{I O C, L C}=I B-R-G_{H G}
$$

Sustituyendo la ecuación 16 en 17, tomando en cuenta las ecuaciones 1 y 3 para que los porcentajes o fracciones queden claramente explicitados a la derecha de la ecuación, la ecuación se sintetiza como la siguiente:

$$
I_{I O C, L C}=(1-\omega)(1-\alpha) P Q
$$

La IOC asume la totalidad de los costos operativos $O P E X$, por lo tanto su utilidad operativa se reduce a:

$$
U O_{I O C, L C}=(1-\omega)(1-\alpha) P Q-O P E X
$$

La tasa de impuestos $\mu$ sobre su utilidad operativa es:

$$
T_{L C}=\mu U O_{I O C, L C}
$$

Sustituyendo la ecuación 18 en la 20 se obtiene:

$$
T_{L C}=\mu[(1-\omega)(1-\alpha) P Q-O P E X]
$$

La utilidad neta de la IOC $U_{I O C}$ resulta al restar los impuestos a su utilidad operativa:

$$
U_{I O C}=U O_{I O C}-T_{L C}
$$

Sustituyendo las ecuaciones 18 y 21 en 22 resulta en un segundo equilibrio:

$$
* * U_{I O C}=(1-\mu)[(1-\omega)(1-\alpha) P Q-O P E X]
$$


Tómese la anterior ecuación como el segundo tipo de equilibrio de este contrato LC y denótese con 2 asteriscos $(* *)$.

\section{Contratos de servicios de riesgo (con recompra)}

El ingreso bruto $I_{B}$ se obtiene igual que en los modelos anteriores (ver ecuación 1).

Los costos operativos $O P E X$ se deben restar del ingreso bruto y un porcentaje $\varphi$ de lo restante determina el ingreso del gobierno o $\mathrm{HG} G_{H G, B B A}$

$$
G_{H G, B B A}=\varphi\left(I_{B}-O P E X\right)
$$

Sustituyendo la ecuación 1 en 24, el ingreso del gobierno resulta en:

$$
G_{H G, B B A}=\varphi(P Q-O P E X)
$$

Por su parte, la utilidad operativa de la IOC $U O_{I O C}$ queda expresada de la siguiente manera:

$$
U O_{I O C, B B A}=(1-\varphi)(P Q-O P E X)
$$

Este pago a la IOC incluye la amortización de la inversión $\beta C A P E X$, el pago de cargos bancarios $C B$ y la remuneración a la IOC que en este caso equivale a la utilidad neta de la IOC $U_{I O C, B B A}$, la cual se reduce al tercer equilibrio como sigue:

$$
* * * U_{I O C, B B A}=I_{I O C, B B A}-\beta C A P E X-C B
$$

Tómese finalmente a este equilibro del contrato de BBA con 3 asteriscos $(* * *)$.

Donde $\beta$ es depreciación. Los ingresos de la IOC son libres de impuestos. Obsérvese que los modelos asumen resultados sin diferencias entre tiempos de exploración, extracción y producción, es decir, son juegos simultáneos en un solo momento en el tiempo.

La definición de porcentajes es entonces como sigue:

$\alpha$ es el porcentaje de regalías;

$\omega, \delta$ se definen como porcentajes de reparto de la utilidad o la producción sobre ingresos brutos (LC) o sobre utilidad operativa (PSC), correspondientes a IOC;

$\sigma$ es el máximo porcentaje de los ingresos reconocible para la recuperación de costos o cost oil en contratos PSC;

$\mu$ es el impuesto corporativo sobre la renta;

$\beta$ es el porcentaje de depreciación de capital en CAPEX; y finalmente

$\varphi$ es el porcentaje de los ingresos correspondiente al HG, y (1- $\delta$ ) es el remanente para la IOC.

Con lo anterior, es claro que los contratos BBA son inferiores a los LC y los de producción compartida o PSC, tanto para el gobierno como para la contratista, ya que $\varphi(P Q-O P E X)<\omega \mu$ $P Q$ entre BBA y LC. Igualmente para el contratista, $* * *<* *$.

Ahora, entre PSC y LC, para el gobierno LC podría ser más ventajoso que PSC, pero no así para la IOC. Es decir, puede asumirse que $\omega \mu \alpha P Q>\mu \alpha(1-\sigma)(1-\delta) P Q$. Por el lado de la IOC, $*{ }^{* *}$, pero condicionado a que el porcentaje de reparto sea alto, así como que el porcentaje de cost oil de recuperación sea atractivo. Es decir, deben asumirse los anteriores porcentajes para que claramente se tenga del presente análisis que $*>* *>* * *$ para la IOC.

De manera visual podrían resumirse los planteamientos desarrollados como se expresa en la figura 1.

El análisis empírico se realiza en la próxima sección. 


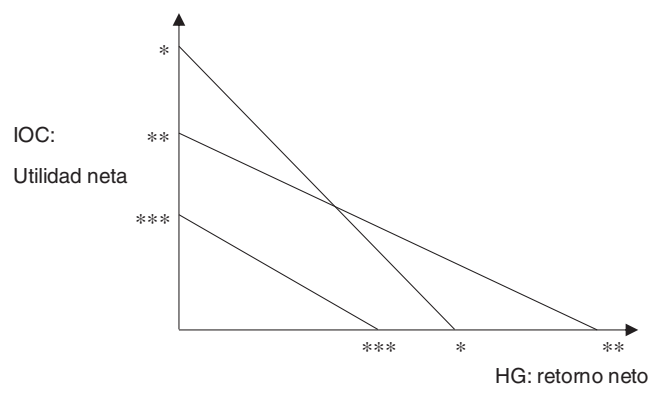

Figura 1. Parece claro que para la IOC los contratos tipo (*) serán totalmente preferibles a los LC (**), y ordenadamente los mismos preferibles a BBA (***). Sin embargo, a pesar de que para el gobierno o HG *** es dominado por los demás, existe un nivel tal que * es preferible a ** y en otros casos de baja utilidad neta para la IOC, ** es preferible a $*$. Los tipos de contrato serán preferibles ahora por el tipo de empresas contratistas: globales o especializadas.

Fuente: elaboración propia.

\section{Conducta y desempeño de empresas: análisis de panel de datos}

No existe información detallada y suficiente de todos los contratos a nivel mundial, ni un universo conocido de empresas especializadas y activas en contratos de E\&E, ya que muchas no están listadas en mercados accionarios y no toda la actividad de E\&E es mediante contratos como los descritos, porque muchos países petroleros mantienen la propiedad gubernamental en sus propias NOC. Igualmente, pueden existir empresas que acepten varios tipos de contratos de E\&E dependiendo de los gobiernos anfitriones. Sin embargo, usando especificaciones alternativas de análisis de panel de datos, puede comprobarse parte de las hipótesis del presente estudio, como se presenta a continuación.

Se busca aislar a las empresas por origen y nivel de cobertura, concentrándonos entre las llamadas empresas globales y las especializadas en E\&E solamente, así como sus variables de desempeño financiero, tamaño mediante la variable de activos, para acercarse a la tendencia de tipo de contratos, ya que no es posible realizar análisis de riesgo accionario o rendimientos medidos en mercados financieros (OECD, 2012).

Las empresas podrían separarse por subsector dentro de la cadena de hidrocarburos. Usando la clasificación de un estudio de Mittal, Dholakia, Han y Dayal, 2015, para empresas de los EE. UU., es notorio que se trata de un mercado de alta tecnología, con grandes requerimientos de capital y financiamiento, y por ende, un sector de empresas concentrado y de tipo oligopolio (Mittal et al., 2015). Las empresas contratistas son las siguientes (tabla 1).

$$
\hat{y}_{i t}=w_{i t} \hat{\theta}+\hat{u}_{i t}
$$

El siguiente análisis de validación de la teoría expuesta se concentra en las primeras 2 clasificaciones y donde existen datos económico-financieros de 17 empresas descritas en los períodos 2005-2015. Se trata de 8 empresas globales y 9 especializadas de E\&E, usando información estandarizada de Thomson Reuters y de Bloomberg (2016). La capacidad de ingresos sobre activos de las grandes globales y los retornos sobre ingresos netos o ROE son las variables dependientes. El modelo de panel podría especificarse de manera general como sigue:

$$
\hat{y}_{i t}=w_{i t} \widehat{\theta}+\hat{u}_{i t}
$$


Tabla 1

Clasificación de empresas petroleras por subsector

IOC integradas (las globales)

IOC especializadas en E\&E

Midstream oil and gas

IOC especializadas solo en servicios y construcción y mantenimiento
BP, Chevron, Conoco Phillips, Exxon Mobil, Shell, Statoil, Total, ENI

Anadarko Petroleum, Apache, EOG

Resources, Hess, Marathon Oil, Murphy Oil, Newfield Exploration, Noble

Energy, and Occidental Petroleum

Buckeye Partners, Enbridge Energy

Partners, Enterprise Products Partners, Genesis Energy, Kinder Morgan, Plains

All American Pipeline, Spectra Energy, Sunoco, Targa Resources Baker Hughes, Cameron, Dresser-Rand,

FMC Technologies, GE Oil \& Gas, Halliburton, Hercules Offshore, MRC Global,

National Oilwell Varco, Newpark Resources, Oceaneering, Oil

States

International, Parker Drilling, Schlumberger, Transocean and

Weatherford

Fuente: elaborado por los autores con datos de Mittal et al. (2015).

Donde los estimadores corresponden a transformaciones alternativas para est yit, wit y donde uit de yit y w'it = [1 x'it], uit. En esta especificación est yit es una función conocida de solo yi $1, \ldots$, $y i T$, y de manera similar se da la misma estructura para est wit y est uit.

Los modelos en panel deben ajustarse por problemas de eficiencia estadística de posible heteroscedasticidad y correlación seriada de errores de estimación. Asimismo, para estimadores consistentes se estiman modelos con efectos fijos o dummies por tipo de empresa global versus especializada, en estimaciones del panel equilibrado de1) pooled OLS o POLS; 2) WLS basada en varianzas de los errores para consistencia; 3) panel con datos desmediados o Within Panel; 4) panel en primeras diferencias temporales o first diff; y 5) transformaciones GLS de tipo Koyck o random effects GLS o RE-GLS (Cameron y Trivedi, 2005).

De la especificación general anterior, POLS implica que $\theta=\left[\alpha \beta^{\prime}\right]^{\prime}$. Para modelos de panel con datos desmediados, est $y_{i t}=\left(y_{i t}-\mu \bar{y}_{i}\right)$ y $\theta$ es igual a los coeficientes de los regresores que cambian en el tiempo. Asimismo, el modelo de panel en primeras diferencias se especifica con el caso especial del modelo descrito arriba, para first diff. Finalmente, el caso de RE- GLS, est $y_{i t}=\left(y_{i t}-\mu \bar{y}_{i}\right), w, w e s t w_{i t}^{\prime}=\left(w_{i t}-\mu \bar{w}_{i}\right)$.

Los resultados aparecen en la tabla 2, y los resultados econométricos en las tablas 3 y 4:

Los modelos están separados en 2 de acuerdo con las variables dependientes ROE por un lado, e ingresos netos de operación por el otro. Una variable de control es el tamaño medido por el valor de activos, el cual en las primeras estimaciones no parece ser significativo, e incluso presenta signo negativo. Por otra parte, parece claro que la variable binaria que separa a las empresas globales (diversificadas) de las especializadas es altamente significativo en el primer grupo de análisis de panel, mostrando que las globales, al estar más diversificadas, tenderán a buscar contratos de relativo mayor riesgo y mayor inversión, o más government take. Los modelos GLS, y fist differences son claramente más consistentes que los previos de POLS y WLS, de acuerdo con la teoría. El análisis de panel agrupa casi de manera simétrica a 8 empresas globales y 9 especializadas en 11 años de la dimensión temporal. La variable dummy toma el número 1 para globales y 0 para especializadas. 
Tabla 2

Estadísticos principales, usando las observaciones 1:01-17:11, muestra de 17 empresas entre 2005 y 2015. Los datos monetarios están en millones de dólares (USD)

\begin{tabular}{|c|c|c|c|c|c|}
\hline Variable & Media & Mediana & Mínimo & & Máximo \\
\hline roe & 12.3187 & 15.3091 & -162.221 & & 41.2868 \\
\hline netincome & 7675.64 & 3486.94 & -23119.0 & & 45220.0 \\
\hline assetsize & 111674. & 56259.0 & 4768.00 & & 357512 . \\
\hline globalspec & 0.470588 & 0.00000 & 0.00000 & & 1.00000 \\
\hline USEU & 0.705882 & 1.00000 & 0.00000 & & 1.00000 \\
\hline Variable & Desv. típica. & C.V. & Asimetría & \multicolumn{2}{|r|}{ Exc. de curtosis } \\
\hline roe & 21.9379 & 1.78086 & -4.35113 & \multicolumn{2}{|r|}{28.4596} \\
\hline netincome & 11050.9 & 1.43973 & 1.14447 & \multicolumn{2}{|r|}{1.62724} \\
\hline assetsize & 103803. & 0.929514 & 0.824450 & \multicolumn{2}{|r|}{-0.568041} \\
\hline globalspec & 0.500474 & 1.06351 & 0.117851 & \multicolumn{2}{|r|}{-1.98611} \\
\hline USEU & 0.456868 & 0.647230 & -0.903696 & \multicolumn{2}{|r|}{-1.18333} \\
\hline Variable & Porc. 5\% & Porc. $95 \%$ & Rango IQ & & vaciones ausentes \\
\hline roe & -21.4355 & 33.9570 & 14.9624 & 0 & \\
\hline netincome & -4485.91 & 31165.8 & 12682.5 & 0 & \\
\hline assetsize & 7597.73 & 332698. & 158661. & 0 & \\
\hline globalspec & 0.00000 & 1.00000 & 1.00000 & 0 & \\
\hline USEU & 0.00000 & 1.00000 & 1.00000 & 0 & \\
\hline
\end{tabular}

Nota: generación propia de la base de datos con el paquete estadístico GRETL.

Tabla 3

Análisis de datos en panel: variable dependiente ROE Obs: 187; j=17 (8 empresas globales y 9 especializadas en una sola muestra); $\mathrm{t}=11(2005-2015)$

\begin{tabular}{|c|c|c|c|c|c|c|}
\hline & $\mathrm{C}$ & ASSETSIZE & GLOBAL/SPEC & ADJ R ${ }^{2}$ & LOGLIK & AV. ROE \\
\hline $\begin{array}{l}\text { MOD 1: OLS corregido } \\
\text { por heteroscedasticidad } \\
\text { después de prueba White }\end{array}$ & $\begin{array}{l}8.5375^{* * * *} \\
(3.65)\end{array}$ & $\begin{array}{l}-9.904 \mathrm{e}^{-06} \\
(0.48)\end{array}$ & $\begin{array}{l}10.7924^{* * *} \\
(2.35)\end{array}$ & 0.0486 & -439.575 & 12.32 \\
\hline $\begin{array}{l}\text { MOD 2: WLS basado en } \\
\text { varianzas de errores }\end{array}$ & $\begin{array}{l}12.9187^{* * *} \\
(7.37)\end{array}$ & $\begin{array}{l}-3.565 \mathrm{e}^{-05 * *} \\
(2.52)\end{array}$ & $\begin{array}{l}12.587^{* * *} \\
(3.76)\end{array}$ & 0.0643 & -260.847 & \\
\hline MOD3: GLS datos & $0.0002^{*}$ & $-0.00013^{\text {** }}$ & $10.792^{* *}$ & 0.161 LSDV-825.949 & & \\
\hline Desmediados. & $(1.78)$ & $(2.67)$ & $(2.36)$ & 0.030 Intra & & \\
\hline $\begin{array}{l}\text { MOD4: FIRST DIFF } \\
\text { en } t\end{array}$ & $\begin{array}{l}-7.997^{* * *} \\
(4.95)\end{array}$ & $\begin{array}{l}5.067 \mathrm{e}^{-04 * * *} \\
(4.264)\end{array}$ & $\begin{array}{l}2.00^{* * *} \\
(2.38)\end{array}$ & 0.107 Intra-740.597 & & \\
\hline
\end{tabular}

ASSETSIZE: en monto de activos en millones de USD; GLOBAL/SPEC: dummy, donde 1 asignada a global y 0 a especializada; ROE: retorno sobre capital.

Datos originales generados de Bloomberg y Thompson-Reuters en dólares de los EE. UU. En las estimaciones los errores estándar son robustos frente a heteroscedasticidad y correlación seriada.

\footnotetext{
* Variables significativas $10 \%$.

** Variables significativas a 5\%.

*** Variables significativas a $1 \%$.
} 
Tabla 4

Análisis de datos en panel: variable dependiente: Net Income Obs: 187; j= 17 (8 empresas globales y 9 especializadas en una sola muestra); $\mathrm{t}=11(2005-2015)$

\begin{tabular}{|c|c|c|c|c|c|c|c|}
\hline & $\mathrm{C}$ & ASSETSIZE & GLOBAL/SPEC & & ADJ R ${ }^{2}$ & LOGLIK & AV. NI \\
\hline $\begin{array}{l}\text { MOD 1: OLS corregido } \\
\text { por heteroscedasticidad } \\
\text { después de prueba White }\end{array}$ & $\begin{array}{l}-661.732 \\
(1.643)\end{array}$ & $\begin{array}{l}0.0622^{\text {*** }} \\
(5.089)\end{array}$ & $\begin{array}{l}2209.95 \\
(1.208)\end{array}$ & & 0.3400 & -448.606 & 7675.04 \\
\hline $\begin{array}{l}\text { MOD 2: WLS basado en } \\
\text { varianzas de errores }\end{array}$ & $\begin{array}{l}-329.958 \\
(1.394)\end{array}$ & $\begin{array}{l}0.0575^{\text {**** }} \\
(6.517)\end{array}$ & $\begin{array}{l}2034.77 \\
(1.278)\end{array}$ & & 0.5164 & -259.746 & \\
\hline $\begin{array}{l}\text { MOD3: GLS datos } \\
\text { desmediados }\end{array}$ & $\begin{array}{l}-1393.33^{* * *} \\
(3.237)\end{array}$ & $\begin{array}{l}0.0622^{* * *} \\
(5.080)\end{array}$ & $\begin{array}{l}2209.95^{* *} \\
(2.208)\end{array}$ & & 0.3401 & -448.666 & \\
\hline $\begin{array}{l}\text { MOD4: FIRST DIFF } \\
\text { en } t\end{array}$ & $\begin{array}{l}-2380.36^{* * *} \\
(4.95)\end{array}$ & $\begin{array}{l}0.2564^{\text {**** }} \\
(4.264)\end{array}$ & $\begin{array}{l}2156.80^{* *} \\
(2.38)\end{array}$ & & 0.2541 & -1711.316 & \\
\hline MOD5: RE-GLS & $\begin{array}{l}1389.24^{* * *} \\
(7.619)\end{array}$ & $\begin{array}{l}0.1362^{* *} \\
(4.306)\end{array}$ & $\begin{array}{l}1448.9^{* * * *} \\
10.74)\end{array}$ & $\begin{array}{l}0.631(\mathrm{NI}-1)^{* * * *} \\
(8.36)\end{array}$ & 0.6381 & -363.929 & \\
\hline
\end{tabular}

Datos originales generados de Bloomberg y Thompson-Reuters en dólares de los EE.UU. El modelo 5 hace uso de primeras diferencias, donde la variable dependiente rezagada un período se presenta como variable explicativa, como en Cameron y Trivedi (2005). En las estimaciones los errores estándar son robustos frente a heteroscedasticidad y correlación seriada.

* Variables significativas $10 \%$.

*** Variables significativas a $5 \%$.

*** Variables significativas a $1 \%$.

Por su parte, la tabla 4 presenta la variable de desempeño como ingresos netos, siendo las variables explicativas las mismas que en la tabla 2. Una última especificación del análisis de panel en este caso de flujos monetarios es el modelo RE-GLS. Una vez más, los modelos consistentes, GLS y First Differences, así como el referido RE-GLS son superiores a los primeros POLS y WLS, de acuerdo con la teoría (pruebas de Hausman para la diferencia de parámetros y diferencias de especificación se muestran en resumen en los diferentes cocientes de log-likelihood hacia efectos fijos). Destaca la significación estadística ahora, tanto del tamaño de empresa como su posición global o especializada, comprobándose la hipótesis derivada del modelo teórico de que los patrones de conducta vía tipo de contratos se vinculan con los tipos de empresas, y su desempeño.

Cabe aclarar que la variable de precios internacionales de petróleo, aunque podría asumirse muy importante para los contratos, solo opera con una drástica caída a partir de 2014 en 2 años del panel completo, por lo que es justificado no incluirla en el presente análisis. Una prueba que no se muestra aquí es contrastar la batería de modelos de panel que terminarán en 2013 frente a los modelos que terminan en 2015. Se prefiere contar con más datos a introducir dichas variaciones en precios internacionales en tan pocos períodos. Tal vez ante más observaciones anuales en el futuro podría valorarse dicha caída de precios.

Finalmente, al hacer una revisión de todos los indicadores económico-financieros sobre los márgenes netos, el análisis tabular de la base de datos original de las 17 empresas muestra que en 2005-2007 las especializadas tenían márgenes muy superiores que las globales (de más de $30 \%$ frente a promedios de $10 \%$ de las globales), pero en los últimos años se han deteriorado dramáticamente.

Es notorio que empresas tales como Chevron, Exxon y Total mostraron márgenes positivos, mientras que el resto tuvo mal desempeño sobre todo en 2015. Sin embargo, el desempeño reciente de todas las especializadas en general es peor de manera reciente que las globales, de márgenes muy superiores como se indica hace una década. Todas las especializadas tuvieron márgenes netos negativos en 2015, de un promedio de las 9 especializadas, del 24\% en 2005 (y 11\% en 2014). Esta información parte de la base de datos original. 


\section{Implicaciones y conclusión}

Aunque no es posible llevar a cabo un análisis econométrico de valor de activos con datos de alta frecuencia, parece claro que el grupo de empresas globales integradas presenta un mejor desempeño que las especializadas (en E\&E) al utilizar datos de estados financieros de balances, de flujos de efectivo y de cocientes del análisis financiero generalmente utilizados. Una batería de modelos alternativos de análisis de panel: POLS, WLS, primeras diferencias y RE-GLS con 2 variables dependientes de desempeño comprueban las hipótesis del trabajo.

A pesar de la tendencia secular de menor desempeño en el período 2005-2015 en general, los indicadores de ROE e ingresos netos son sustancialmente mejores entre las globales, ya que al estar integradas y/o diversificadas, cubren desempeños menores en alguna parte de la cadena de valor con otras áreas. Por ejemplo, ante el bajo desempeño de extracción y producción de crudo, el bajo precio es ventajoso como ahorro de costos en la parte de midstream o downstream de la industria.

Asimismo, aunque algunas de las firmas especializadas del análisis, como es el caso de Andarko o Marathon, se parecen más a las globales que a sus contrapartes de E\&E, las empresas analizadas en este segmento muestran como grupo menor desempeño en las 3 variables indicadas que las globales.

¿Qué podría concluirse de las preferencias esperadas por tipo de contrato? Parece que empresas con: a) alta tecnología; b) capacidad de inversión financiada y buena reputación financiera; c) con capacidad de cubrir riesgo mediante integración/diversificación estarían orientadas a absorber más riesgos, pero con más retornos, como son los contratos de producción compartida o PSC. En contraste, empresas con menor desempeño e incapacidad de cubrir riesgos preferirán los contratos de licencia, como se muestra claramente de los equilibrios del análisis de patrones de conducta mediante preferencias de contratos PSC o LC.

Para los gobiernos anfitriones un portafolio de opciones de contratos de E\&E parece una solución óptima para el caso de información asimétrica sobre el tipo de conducta estratégica de la empresa buscadora de contratos de E\&E, entre las que están las del presente estudio. Con ello, los gobiernos al ofrecer un grupo de opciones de contratos permitirían la autoselección de compañías y aumentarían el número de empresas solicitantes de contratos. Sin embargo, los contratos de licencia atraerían a más empresas inversionistas cuya aversión al riesgo fuera mayor en ciclos económicos pesimistas, siempre que el conjunto de contratos sea abundante, ya que más incertidumbre por el tipo de proyecto significa menos interés en la inversión o inversiones con retornos más bajos, pero como gobierno anfitrión se desearía la máxima atracción de inversionistas posible. Ello sin considerar cambios en el trato fiscal de los contratos analizados.

Finalmente, parece claro que la tipificación teórica de las características de contratos alternativos de E\&E, como los implantados por la Reforma energética de México, deben seguir bases contractuales similares en el mundo. Un estudio posterior con más datos podrá desglosar todas las variables tanto a nivel macro- como microeconómicas y financieras. Adicionalmente, un HG como en México deberá internalizar el tipo de riesgo/tipo de empresa que deba seleccionar en las siguientes licitaciones y rondas de petróleo en México.

\section{Bibliografía}

Al-Attar, A. y Alomair, O. (2005). Evaluation of upstream petroleum agreements and exploration and production costs, http://dx.doi.org/10.1111/j.1468-0076.2005.00154.x. OPEC Review. Organization of the Petroleum Exporting Countries. 
Ashong, M. (2010). Cost recovery in prdocution sharing contracts: Opportunity for striking it rich or just another risk not worth bearing. Dundee, United Kingdom: University of Dundee-Centre for Energy, Petroleum and Mineral Law Policy.

Blake, J. y Roberts, M. (2006). Comparing petroleum fiscal regimes under oil price uncertainty. Resources Policy, 31 , 95-105. http://dx.doi.org/10.1016/j.resourpol.2006.08.001

Cameron, A. C. y Trivedi, P. K. (2005). Microeconometrics: Methods and applications. New York: Cambridge University Press.

Diario Oficial de la Federación DOF (20 diciembre de 2013). DECRETO por el que se reforman y adicionan diversas disposiciones de la Constitución Política de los Estados Unidos Mexicanos, en Materia de Energía. Ciudad de México, MX: Diario Oficial de la Federación. Disponible en: http://www.dof.gob.mx/nota_detalle.php?codigo= $5327463 \&$ fecha=20/12/2013 consultado 2 mayo 2016.

EITI (2016). El Estándar del EITI, 2016. Oslo: Secretaría Internacional del EITI. Disponible en: https://eiti.org/ files/spanish_eiti_standard_0.pdf consultado 2 febrero 2016

Feng, Z., Zang, S. B. y Gao, Y. (2014). On oil investment and production: A comparison of production sharing contracts and buyback contracts. Energy Economics, 42, 395-402. http://dx.doi.org/10.1016/j.eneco.2014.01.010

Ghandi, A. y Lin, C. (2014). Oil and Gas Service Contracts around the World: A Review. Energy Strategy Reviews, 3, 63-71. http://dx.doi.org/10.10161/j.esr 2014.03.001

Grunstein, M. (2010). De la caverna al mercado una vuelta al mundo de las negociaciones petroleras. Ciudad de México, D.F: CIDAC-Felou.

Isehunwa, S. y Uzoalor, E. I. (2011). Evaluation of true government take under fixed and sliding royalty scales in Nigerian oil industry. Australian Journal of Basic and Applied Sciences, 5, 735-741.

Jaffe, A. M. y Soligo, R. (2007). The International Oil Companies. Houston: The James A. Baker III Institute for Public Policy; Rice University.

Jenik, R. (2005). The ABC's of petroleum contracts: License-concession agreements joint ventures and production-sharing agreements. En S. Tsalik y A. Schiffrin (Eds.), Covering oil: A reporters guide to energy and development (pp. 61-99). Open Society.

Johnston, D. (1994). International petroleum: Fiscal systems and production-sharing contracts. Tulsa, United States: PennWell Publishing Corporation.

Johnston, D. (2003). International exploration economics, risk and contract analysis. Oklahoma, United States: PenWell.

King y Spalding, L. L. P. (2005). An introduction to upstream government petroleum contracts: Their evolution and current use. OGEL Journal of Production Sharing Contracts.,. Disponible en: https://www.ogel.org/article.asp?key=1730.

Laffont, J. (2005). Regulation and development. Cambridge: Cambridge University Press.

Le Leuch, H. (1988). Contractual flexibility in new petroleum investment contracts. En B. N. Walde (Ed.), Petroleum investment policies in developing countries. London, United Kingdom: Graham and Trotman.

Mittal, V., Dholakia, U., Han, K., Dayal, A. (2015). Sector-level results from the 2015 strategy and corporate performance in the energy industry (SCOPE) Study. Houston: Rice University JGSB Energy Industry Initiatives Report [consultado 20 Nov 2016]. Disponible en: https://papers.ssrn.com/sol3/papers.cfm?abstract_id=2664064.

OECD. (2012). Getting it right: una agenda estratégica para las reformas en México. OECD Publishing. Disponible en: http://www.oecd.org/centrodemexico/Getting\%20It\%20Right\%20EBOOK.pdf consultado 2 febrero 2016

Van Groenendaal, W. J. y Mazraati, M. (2006). A critical review of Iran's buyback contracts. Energy Policy, 34(18), 3709-3718. http://dx.doi.org/10.1016/j.enpol.2005.08.011 